\title{
Improved ant colony algorithm for path planning of fixed wing unmanned aerial vehicle
}

\author{
Hongchao Zhao ${ }^{1 *}$, and Jianzhong Zhao ${ }^{2}$ \\ ${ }^{1}$ Department of Automation, Yantai Institute of Technology, Yantai, Shandong, 264005, China \\ ${ }^{2}$ Coastal Defence College, Naval Aviation University, Yantai, Shandong, 264001, China
}

\begin{abstract}
Aiming at the problems of long search time and local optimal solution of ant colony algorithm (ACA) in the path planning of unmanned aerial vehicle (UAV), an improved ant colony algorithm (IACA) was proposed from the aspects of simplicity and effectiveness. The flight performance constraints of fixed wing UAVs were treated as conditions of judging whether the candidate expanded nodes are feasible, thus the feasible nodes' number was reduced and the search efficiency was effectively raised. In order to overcome the problem of local optimal solution, the pheromone update rule is improved by combining local pheromone update and global pheromone update. The heuristic function was improved by integrating the distance heuristic factor with the safety heuristic factor, and it enhanced the UAV flight safety performance. The transfer probability was improved to increase the IACA search speed. Simulation results show that the proposed IACA possesses stronger global search ability and higher practicability than the former IACA.
\end{abstract}

Keywords: UAV, Path planning, IACA, Pheromone, Heuristic function.

\section{Introduction}

Due to their agility and good ability, unmanned aerial vehicles (UAVs) are widely used in both military and civilian fields. Path planning problem is an important issue of UAV system, thus it is paid much attention to by many researchers. The UAVs usually execute their tasks in three-dimensional (3D) complex wide space environment, so the path planning algorithm is required to balance computing complexity, search time, and path cost. There are many kinds of path planning algorithms, which can be divided into two categories: conventional classical algorithms and modern intelligent algorithms [1]. The conventional classical algorithms include Dijkstar algorithm, simulated annealing algorithm, Dubins curve, artificial potential field, rapidly exploring random tree and its modified algorithms. The modern intelligent algorithms include $A^{*}$ search algorithm, genetic algorithm (GA), particle swarm algorithm (PSO), ant colony algorithm (ACA), pigeon-inspired optimization (PIO) algorithm, etc.

A lot of scholars focus on ACA for it has some advantages such as positive feedback, distributed computing and heuristic search. However it has the disadvantages of long search

\footnotetext{
${ }^{*}$ Corresponding author: zhaohongchao@yitsd.edu.cn
} 
time and local optimal solution. Some improved schemes are presented by scholars, such as multi-colony ant optimization [2], hybrid algorithm of ACA and GA [3], cellular ant colony algorithm [4], combining ACA with artificial potential field [5, 6], improved chaotic ant colony algorithm [7], quantum ant colony algorithm [8], etc. Both local pheromone and global pheromone were updated, and heuristic function was also modified in [9, 10], which improved the global search ability of ACA. The weighted sort pheromone update rule was adopted, and the state transfer probability was also modified in [11-13]. Xu et al. [14] adopted adaptive parameters adjustment strategy, updated both local pheromone and global pheromone, and introduced disturbance factor to the state transfer probability, so that the convergence speed was increased. Ouyang et al. [15] imported threat effect to pheromone update rule and improved the heuristic function, which increased the speed performance and security of path planning. Considering the calculating ability of the UAV's computer isn't high, we further improve the ACA from the aspects of simplicity and effectiveness.

The rest of this paper is organized as follows. The UAV flight environment is described and the flight performance constraints of fixed wing UAVs are analyzed in Section 2. In Section 3, some effective improved schemes are presented to overcome the disadvantages of the conventional ACA. Simulation results are discussed in Section 4. Finally, Section 5 concludes this paper.

\section{Description of UAV flight environment}

It is researched in this paper that a fixed wing UAV executes the natural disaster reconnaissance task in the mountain area. There exist some obstacles in the task environment, such as peaks, electric wires, and trees. Moreover, there exist some wind shear areas around the mountain peaks. The mountain peaks are simulated by cones, and the mountain terrain altitude is expressed as

$$
z_{1}(x, y)=z_{0}+\sum_{m=1}^{M} h_{m} \exp \left[-\left(\frac{x-x_{m}}{x_{s m}}\right)^{2}-\left(\frac{y-y_{m}}{y_{s m}}\right)^{2}\right]
$$

where $z_{0}$ is the basic terrain altitude, $M$ the total number of peaks, $h_{m}$ the height of the $m$ th peak, $\left(x_{m}, y_{m}\right)$ the ground coordinate of the $m$ th peak center, $x_{s m}, y_{s m}$ the peak gradient in $x$ and $y$ orientation, respectively.

The wind shear areas are expressed as cylinders. The ground center coordinate of the $m$ th wind shear area is $\left(x_{w m}, y_{w m}\right)$, its radius $r_{m}$, and its height $h_{m}^{\prime}$. Considering the threats of electric wires and trees, the UAV flight height must be much higher than their maximum height for the sake of flight safety. The 3D environment space is discretized to a grid map. The positions of all obstacles and threats are supposed to be known beforehand, and the grids occupied by them can't be passed by UAV.

The problem of UAV path planning generally refers to the process to search a flight path from the start point to the goal point, which satisfies the demands of flying range, energy, threat avoidance, and flight performance constraints. We have already researched the flight performance constraints of UAV in [16], which need to be satisfied in the path planning. In this paper the flight performance constraints are treated as conditions of judging whether the candidate expanded nodes are feasible. If a candidate expanded node satisfies all the constraints, it is a feasible node and joins the allowed transition node set; or it is rejected. So the feasible nodes' number is reduced. Moreover, we adopt a direct setting method for the height planning of $3 \mathrm{D}$ path planning, thus the $3 \mathrm{D}$ path planning is simplified to the two- 
dimensional (2D) path planning [16]. The IACA searches candidate expanded nodes only in the 2D plane grids, so the search efficiency is effectively raised.

\section{Design of the IACA}

We propose some effective improved schemes on the basis of the former improved methods, in order to overcome the disadvantages of the conventional ACA.

\subsection{Improved pheromone update rule}

In the conventional ACA the initial pheromone distributes evenly, and the attraction degree of the goal point isn't outstanding, so the probability of ants finding the goal point is low. In order to overcome this defect and to enhance the attraction degree, the initial pheromone of the goal point is set a big value, whereas the initial pheromones of other grid nodes in the task space are set small values. The pheromone update rule is improved by combining local pheromone update and global pheromone update.

(1) Local pheromone update rule

The pheromone concentration of a node should be reduced after an ant passes this node so as to avoid repeated search. It increases the chance of ant colony finding more paths and avoids the local optimal problem. The local pheromone update rule is given as follows,

$$
\tau_{i j}=(1-\xi) \tau_{i j}
$$

where $\tau_{i j}$ is the pheromone concentration in the path segment $(i, j), \xi$ is the pheromone decay coefficient, $0<\xi<1$.

(2) Global pheromone update rule

After all ants complete their own paths, the ant colony complete once iteration, then the pheromones of all the environment nodes are updated based on the paths traveled by all ants. In the former research results only the nodes in the optimal path of this iteration are added the pheromone concentration [8, 10, 13]. This update rule expands the pheromone concentration difference between the optimal path and other paths and increases the search speed, but it easily causes IACA to fall into the local optimal problem. The improved strategy in this paper is not only to add the pheromones of all paths but also to add again the pheromones of the optimal path. The objective is not only to ensure the pheromone concentration difference between the optimal path and other paths but also to maintain the pheromone concentration of other paths, and hence the variety of planning paths is enhanced and the problem of local optimal solution is overcome. The global pheromone update rule is given as follows,

$$
\tau_{i j}(t+1)=(1-\rho) \tau_{i j}(t)+\rho\left(\Delta \tau_{i j}+\Delta \tau_{i j}^{*}\right)
$$

where $t$ represents the $t$ th iteration, $\rho$ is the pheromone volatility factor, $0<\rho<1, \Delta \tau_{i j}$ is the pheromone increment in the path segment $(i, j)$ added by the ant colony, $\Delta \tau_{i j}^{*}$ is the pheromone increment in the path segment $(i, j)$ of the optimal path.

$$
\begin{gathered}
\Delta \tau_{i j}=\sum_{n=1}^{N} \Delta \tau_{i j}^{n} \\
\Delta \tau_{i j}^{n}= \begin{cases}Q / L_{n}, & \text { the } n \text {th path passes }(i, j) \\
0, & \text { otherwise }\end{cases}
\end{gathered}
$$




$$
\Delta \tau_{i j}^{*}=\left\{\begin{array}{cl}
Q / L_{\text {best }}, & \text { the optimal path passes }(i, j) \\
0, & \text { otherwise }
\end{array}\right.
$$

where $N$ is the total number of ants, $Q$ is a pheromone strength constant, $L_{n}$ is the length of the $n$th path, $L_{\text {best }}$ is the length of the optimal path in the $t$ th iteration.

The pheromone concentrations of all the paths are limited into the range $\left[\tau_{\min }, \tau_{\max }\right]$, which can prevent that the pheromone concentrations of some paths are large enough to cause search stagnation.

\subsection{Improved heuristic function}

The heuristic function is an important factor affecting the search speed and algorithm stability. The conventional heuristic function can't well guide an ant to move to the goal point, and it can't also guide an ant to avoid the threats in the environment. In [10] the safety heuristic factor, the distance heuristic factor, and the path offset factor are added to the heuristic function, where the distance heuristic factor is designed as follows,

$$
D_{j}=w d_{i j}+(1-w) d_{j G}
$$

where $d_{i j}$ is the length of the path segment $(i, j), w$ is a weight, $0<w<1, d_{j G}$ is the distance between the $j$ th node and the goal node.

The safety heuristic factor is a kind of threat avoidance strategy. In [10] it is designed according to the heights of candidate nodes, but it doesn't fit the mountain environment with some peaks. Considering the effect of threats such as peaks and wind shear areas, the distance between a candidate node and the threats is farther, the flight safety of an UAV is higher. The safety heuristic factor needs to be modified to enhance the flight safety, and moreover, the path offset factor in [10] isn't necessary.

We take the minimum horizontal distance between a candidate node and all threats as the safety heuristic factor. The ground coordinate of the $j$ th node is $\left(x_{j}, y_{j}\right)$, and its horizontal distance to the $m$ th peak center $\left(x_{m}, y_{m}\right)$ is as follows,

$$
\operatorname{hd}_{j m}=\left[\left(x_{j}-x_{m}\right)^{2}+\left(y_{j}-y_{m}\right)^{2}\right]^{1 / 2}
$$

where $m=1,2, \cdots, M$. Its horizontal distance to the $m$ th wind shear area center $\left(x_{w m}, y_{w m}\right)$ is as follows,

$$
\text { hd } 2_{j m}=\left[\left(x_{j}-x_{w m}\right)^{2}+\left(y_{j}-y_{w m}\right)^{2}\right]^{1 / 2}
$$

The safety heuristic factor of the $j$ th node is as follows,

$$
\mathrm{hf}_{j}=\min \left(\mathrm{hd}_{j m}, \mathrm{hd} 2_{j m}\right)
$$

The improved heuristic function of the $j$ th node is designed as follows, integrating the distance heuristic factor with the safety heuristic factor,

$$
\eta_{i j}(t)=\mathrm{hf}_{j} / D_{j}
$$

\subsection{Improved transfer probability}

The improved transfer probability of the $n$th ant is calculated by 


$$
S_{i j}^{n}(t)= \begin{cases}\arg \max _{\lambda \in \text { allowed }_{n}}\left[\tau_{i \lambda}^{\alpha}(t) \eta_{i \lambda}^{\beta}(t)\right], & q \leq q_{0} \\ P_{i j}^{n}(t), & q>q_{0}\end{cases}
$$

where allowed ${ }_{n}$ is the allowed transfer node set of the $n$th ant in the current node, $\alpha$ and $\beta$ are the importance degree factors of pheromone and heuristic function, respectively, $q$ is a random number, $0 \leq q \leq 1, q_{0}$ is a constant to be set up according to the actual engineering, $P_{i j}^{n}(t)$ is the conventional transfer probability, that is,

$$
P_{i j}^{n}(t)= \begin{cases}\frac{\tau_{i j}^{\alpha}(t) \eta_{i j}^{\beta}(t)}{\sum_{s \in \text { allowed }_{n}} \tau_{i s}^{\alpha}(t) \eta_{i s}^{\beta}(t)}, & j \in \text { allowed }_{n} \\ 0, & \text { otherwise }\end{cases}
$$

The improved transfer probability combines deterministic transfer mode and heuristic transfer mode, and the transition of both is decided by the relation of $q$ and $q_{0}$. It increases the IACA search speed and overcome the disadvantages of long search time of the conventional ACA.

\section{Simulation analysis}

In order to verify the effectiveness of the proposed IACA, numerical simulations are carried out in this section. The simulation model of path planning of a fixed wing UAV is constructed by MATLAB R2010b software. The task environment space is set as $100 \mathrm{~km} * 100 \mathrm{~km} * 4 \mathrm{~km}$. Because we adopt a direct setting method for the height planning, the $3 \mathrm{D}$ path planning is transformed into $2 \mathrm{D}$ path planning. The ground task area is $100 \mathrm{~km} * 100 \mathrm{~km}$, and it is discretized to even grids with size $50 * 50$. The start point of the UAV is $(0,20)$, and the goal point $(80,80)$. There are 8 peaks and 8 wind shear areas in the mountain environment.

Firstly the conventional ACA is used to plan the UAV's path, but it can't find a feasible path, because it doesn't have goal guidance mechanism and safety heuristic factor. Secondly, some former improved ant colony algorithms are used to plan the UAV's path. It is found from several simulation experiments that they can't find a feasible path without goal guidance mechanism and safety heuristic factor. Finally, we compare the IACA in this paper with the former IACA only from the difference of global pheromone update rule, in order to validate the superiority of our IACA. The former global pheromone update rule is to add the pheromone concentration of the optimal path in every iteration $[8,10,13]$. Our global pheromone update rule is not only to add the pheromone concentration of all paths but also to add again the pheromone concentration of the optimal path. The above two algorithms adopt goal guidance mechanism, safety heuristic factor, and big initial pheromone of the goal point. In the simulation experiments the IACA parameters are set as follows, the iteration number $I_{\max }=50$, the total number of ants $N=20, \xi=0.3, \rho=0.3, Q=60$, $\tau_{\min }=0.4, \tau_{\max }=6, w=0.3, \alpha=2.5, \beta=1.5, q_{0}=0.8$. The simulation experiments are executed 100 times by Monte Carlo method. The simulation result statistics show that the average time consuming of path planning is $13.49 \mathrm{~s}$ in our IACA vs. $13.74 \mathrm{~s}$ in the former IACA. The difference of the average time consuming is small. But the length of path planned by our IACA is shorter than that by the former IACA. The contrast of the path length of both algorithms is shown in table 1 . 
Table 1. Contrast of the path length of both algorithms.

\begin{tabular}{lccc}
\hline Algorithms & $\begin{array}{c}\text { Mean value } \\
(\mathrm{km})\end{array}$ & $\begin{array}{c}\text { Maximum } \\
(\mathrm{km})\end{array}$ & $\begin{array}{c}\text { Minimum } \\
(\mathrm{km})\end{array}$ \\
\hline Former IACA & 139.78 & 150.19 & 129.07 \\
Our IACA & 132.25 & 142.69 & 120.42 \\
\hline
\end{tabular}

According to table 1, the mean value of path length of our IACA is $5.39 \%$ smaller than that of the former IACA, the maximum $4.99 \%$, and the minimum $6.70 \%$. The simulation results show that the IACA in this paper possesses stronger global search ability and higher practicability than the former IACA. Five paths are chosen from the paths planed by the former IACA, which are shown in figure 1. Five paths are chosen from the paths planed by the IACA in this paper, which are shown in figure 2.
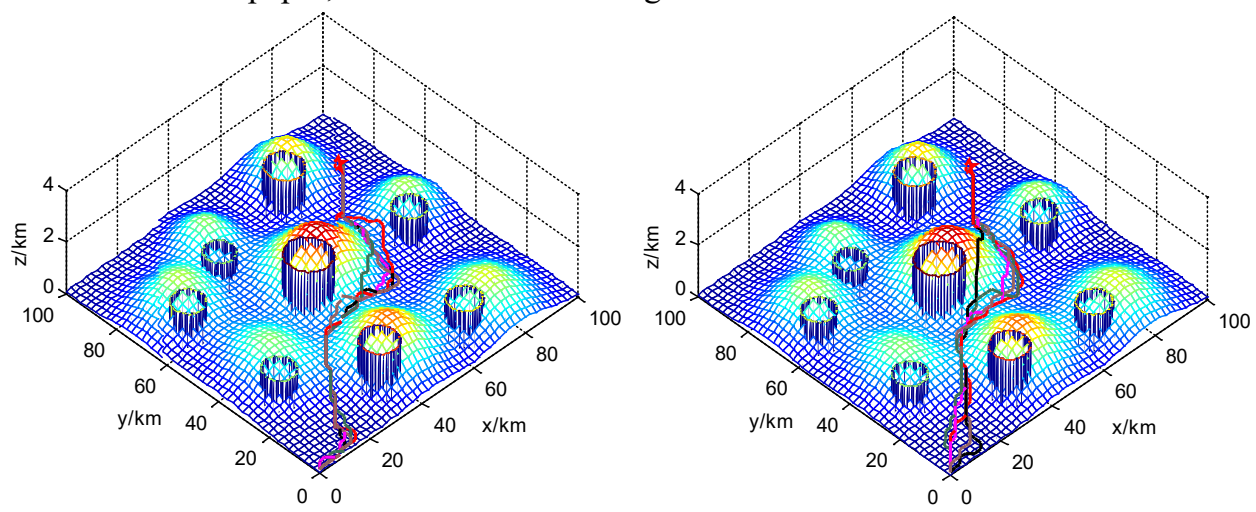

Fig. 1. Five paths planned by the former IACA. Fig. 2. Five paths planned by the IACA in this paper.

\section{Conclusions}

In this paper the improved scheme of ACA is proposed from the aspects of simplicity and effectiveness, in order to overcome the disadvantages of the conventional ACA. The mountain task environment of a fixed wing UAV is described. The flight performance constraints of fixed wing UAVs are treated as conditions of judging whether the candidate expanded nodes are feasible, which reduces the feasible nodes' number and raises the search efficiency. The pheromone update rule is improved to overcome the problem of local optimal solution. The heuristic function is improved by integrating the distance heuristic factor with the safety heuristic factor, which enhances the UAV flight safety performance. The transfer probability is improved to increase the IACA search speed. Simulation results show that the proposed IACA possesses stronger global search ability and higher practicability than the former IACA. In the following work we will analyse dynamic threats in 3D environment space and research the dynamic threat avoidance algorithm in the UAV path planning.

\section{References}

1. Wang, Q., Liu, M., Ren, J., et al. (2019) Overview of common algorithms for UAV path planning. Journal of Jilin University (Information Science Edition), 37: 58-67.

2. Cekmez, U., Ozsiginan, M., Sahingoz, O. K. (2016) Multi-colony ant optimization for UAV path planning with obstacle avoidance, In: 2016 International Conference on Unmanned Aircraft Systems. Arlington. pp. 47-52. 
3. $\mathrm{Hu}, \mathrm{T}$. (2019) Research on 3D surveillance trajectory planning for small UAV based on bio-inspired algorithms. Dissertation of Chongqing University of Posts and Telecommunications, 41-50.

4. Li, X., Wei, R., Zhang, Q., et al. (2017) A path planning for UAVs in urban building areas based on cellular ant colony algorithm. Journal of Air Force Engineering University (Natural Science Edition), 18: 19-23.

5. Xing, D., Zhen, Z., Zhou, C. et al. (2019) Cooperative search of UAV swarm based on ant colony optimization with artificial potential field. Transactions of Nanjing University of Aeronautics and Astronautics, 36: 912-918.

6. Li, X., Ma, R., Zhang, S., et al. (2020) Improved design of ant colony algorithm and its application in path planning. Acta Aeronautica et Astronautica Sinica, 41: 213-219.

7. Liu, R., Yang, F., Zhang, H. (2018) Path planning for UAV based on improved chaotic ant colony algorithm. Command Information System and Technology, 9: 41-48.

8. Xia, G., Han, Z., Zhao, B., et al. (2019) Unmanned surface vessel path planning based on quantum ant colony algorithm. Journal of Harbin Engineering University, 40: 12631268.

9. Jiao, Y. (2019) Research on path planning of UAV based on improved ant colony algorithm. Ship Electronic Engineering, 39: 41-45.

10. Wei, J., Wang, J., Wang, J., et al. (2020) 3D path planning based on improved ant colony algorithm. Computer Engineering and Applications, 56: 217-223.

11. Gao, Y., Chen, X., Zhou, S., et al. (2016) Planning based on improved ant colony algorithm for multiple batches collaborative three-dimensional track. Journal of Northwestern Polytechnical University, 34: 41-46.

12. Jie, D., Tang, X., Chen, J., et al. (2020) Research on conflict resolution technology of multi-UAV based on improved ant colony algorithm. Journal of Wuhan University of Technology (Transportation Science \& Engineering), 44: 141-147.

13. Tang, X., Ji, X., Li, T. (2020) Key technology in multi-UAV conflict detection and resolution strategy. Transactions of Nanjing University of Aeronautics and Astronautics, 37: 175-186.

14. Wu, X., Jia, Y., Zhang, J., et al. (2018) Simulation study of UAV conflict resolution based on an improved ant colony algorithm. Journal of Hebei University of Science and Technology, 39: 166-175.

15. Ouyang, Z., Guo, Q. (2018) Penetration route planning of UAV based on improved ant colony algorithm. Modern Defense Technology, 46: 74-78.

16. Zhao, H., Zhou, H., Wang, S. (2021) Quantum particle swarm optimization algorithm of three-dimensional path planning of unmanned aerial vehicle. Aerospace Control, 39: 4045 . 